\title{
Soft-x-ray resonant magnetic diffuse scattering from strongly coupled $\mathrm{Cu} / \mathrm{Co}$ multilayers
}

\author{
T. P. A. Hase, I. Pape, and B. K. Tanner \\ Department of Physics, University of Durham, South Road, Durham DH1 3LE, United Kingdom \\ H. Dür, * E. Dudzik, and G. van der Laan \\ Daresbury Laboratory, Daresbury, Warrington WA4 4AD, United Kingdom \\ C. H. Marrows and B. J. Hickey \\ Department of Physics and Astronomy, University of Leeds, Leeds LS2 9JT, United Kingdom
}

(Received 22 September 1999)

\begin{abstract}
By separately identifying magnetic and charge scatter, we find conclusive evidence for conformality in magnetic roughness in $\{\mathrm{Co}(8 \AA) \mathrm{Cu}(9 \AA)\}$ multilayers. For layers magnetized in the easy direction, the magnetic roughness equals the structural roughness but increases when magnetized in the hard direction. The in-plane magnetic correlation length, which changes on magnetization, is several orders of magnitude larger than the structural roughness length scales. The magnetic length scale is of the same order as magnetic ripple observed in Lorentz microscopy and is not associated with domains.
\end{abstract}

Giant magnetoresistance (GMR) occurs in multilayers of ferromagnetic layers separated by paramagnetic spacer layers due to the change in the electron scattering cross section when the coupling of adjacent ferromagnetic layers is switched from antiparallel to parallel. Such switching forms the basis of spin-valve structures now used commercially as magnetic data read heads. The spin-dependent scattering appears to occur primarily at the interfaces and to be sensitive to the interface structure. Consequently, the grazing incidence scattering of $x$ rays has been extensively used to determine the morphology of buried interfaces in GMR multilayer systems. ${ }^{1-4}$ Quantitative parameters can be obtained by fitting specific models to the experimental data; ${ }^{5}$ the fitting of both specular and diffuse scatter permits the compositional grading to be distinguished from the true roughness. ${ }^{6}$

The spin-dependent interface scatter believed to give rise to the GMR will be dependent on the chemical roughness of the interface, but may also be related to the roughness of the magnetic moment. As the mean free path for the two spin channels depends on the direction of magnetization, this implies that magnetic disorder at the interfaces may influence the scattering strength. It is far from obvious that the magnetic and structural roughness is always identical, due to the vectorial nature of the magnetization. We can envisage two mechanisms whereby magnetic roughness can arise. The first, in which the moment direction does not change, is directly connected to the chemical composition across the interface and should be correlated with the structural roughness. ${ }^{7}$ A second form of magnetic roughness arises from local rotation of the moments just inside the ferromagnetic material, similar to the magnetic ripple seen in Lorentz micrographs of thin ferromagnetic films. The in-plane length scale of this type of roughness may be expected to be large, due to the magnetostatic energy associated with the moment rotation, and thus will not necessarily correlate to the structural morphology.
There is, therefore, currently great interest in attempts to separately measure chemical and magnetic roughness and their associated in-plane length scales using magnetic scattering of soft $\mathrm{x}$ rays or neutrons. Freeland et $\mathrm{al}^{8}{ }^{8}$ have reported that the magnetic roughness in single CoFe films is always less than the chemical roughness and has a longer in-plane correlation length, supporting earlier measurements on $\mathrm{Co} / \mathrm{Cu} / \mathrm{Co}$ trilayers by McKay et al. ${ }^{9}$ Borchers et al. ${ }^{10}$ using a combination of scanning electron microscopy with polarization analysis and polarized neutron scattering, have shown that the diffuse scattering in weakly coupled $\{$ Co (60 $\AA) \mathrm{Cu}(60 \AA)\}$ multilayers is associated with micrometerscale domains. Although the existence of an off-specular purely magnetic Bragg peak indicates the presence of conformal magnetic roughness, their fitted transverse diffuse scatter measurements are in the transmission geometry and sensitive to domain size only. ${ }^{10}$ The recent specular measurements of Idzerda et al. ${ }^{11}$ on $\mathrm{Co} / \mathrm{Cr} / \mathrm{Co}$ trilayers are also sensitive to domain structure. In this paper we measure separately, from soft-X-ray scattering, chemical and magnetic roughness together with their associated in-plane correlation lengths in conventional, strongly antiferromagnetically coupled $\{\mathrm{Co}(8 \AA) \mathrm{Cu}(9 \AA)\}$ multilayers in which uncorrelated domain walls are not found. Earlier studies ${ }^{8,9}$ which relied on differences in scatter upon field reversal measure a mixture of magnetic and charge scatter. Here, we are able to measure almost pure diffuse magnetic scattering, free from such interference.

The cross section for magnetic x-ray scattering is small, except when resonant enhanced at absorption edges. ${ }^{12}$ Resonant magnetic scattering at the $L$ edges has been performed in the specular condition on $\mathrm{Co} / \mathrm{Cu},{ }^{13} \mathrm{Ag} / \mathrm{Ni},{ }^{14} \mathrm{Fe} / \mathrm{Mn},{ }^{15}$ $\mathrm{Fe} / \mathrm{Co},{ }^{16}$ and $\mathrm{Gd} / \mathrm{Fe} .{ }^{17}$ Resonance enhanced diffuse scattering has been applied to single films and trilayers by McKay et $a l .{ }^{8}$ in which they determined the magnetic scattering by subtraction of data taken in different magnetization states.

The polarization dependence of the magnetic scattering has been reformulated by Hill and McMorrow. ${ }^{18}$ The magnetic scattering amplitude at resonance $f$ depends on the po- 


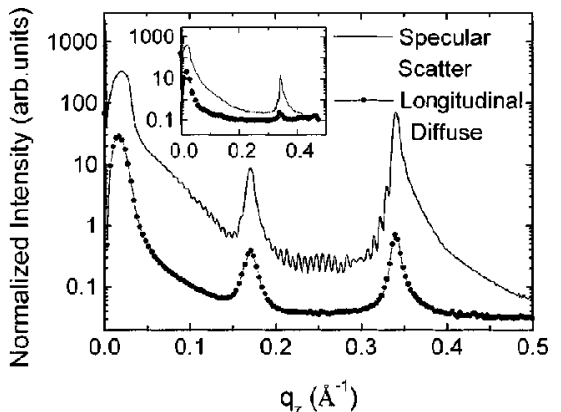

FIG. 1. Specular (line) and longitudinal diffuse scans $\left(-0.3^{\circ}\right.$ offset) (points and line) taken at the Co $L_{3}$ edge for an AF coupled sample with 50 repeats of $\{\mathrm{Co}(8 \AA) / \mathrm{Cu}(9 \AA)\}$ deposited on pure $\mathrm{Si}(001)$. The inset shows same data recorded at the $\mathrm{Cu} L_{3}$ edge.

larization of incident $\mathbf{e}_{0}$ and scattered $\mathbf{e}_{f} \mathrm{x}$ rays, and on the direction of the magnetization vector $\mathbf{M}$ as

$$
f=\left(e_{0} \times e_{f}\right) \cdot \mathbf{M} F,
$$

where $F$ is the resonant response. ${ }^{14}$ Using pure $\sigma$ polarized radiation the magnetic scatter is sensitive to the component of magnetization in the scattering plane. Coherent magnetic scatter will appear at the wave vector of the structural Bragg peak if the interlayer coupling is ferromagnetic, and at half this value if the coupling is antiferromagnetic.

$\mathrm{Co} / \mathrm{Cu}$ multilayers were grown on (001) oriented silicon substrates by magnetron sputtering, incorporating a Meissner trap which allowed a final base pressure in the system of 2 $\times 10^{-8}$ Torr to be realized. ${ }^{19}$ The thickness of $8 \AA$ Co and 9 $\AA \mathrm{Cu}$ was chosen to correspond to the first $\mathrm{AF}$ coupling peak. Two sets of samples were grown, one of 50 repeats on a bare silicon substrate and one of 25 repeats on a thin coating of silicon nitride, the latter for comparison with equivalent samples prepared for Lorentz electron microscopy. Magneto-optical Kerr effect, ${ }^{20}$ magnetization measurements, ${ }^{19}$ and Lorentz microscopy ${ }^{21}$ showed that all were strongly AF coupled. They exhibited up to 60\% GMR at room temperature.

Soft-x-ray scattering experiments were performed on a two-circle diffractometer housed in a high vacuum chamber on stations 1.1 (Ref. 22) and 5U1 (Ref. 23) at the Daresbury SRS. Energies in the range of 200 to $1400 \mathrm{eV}$ with a resolution of $50 \mathrm{meV}$ and flux of typically $10^{10}$ photons $/ \mathrm{sec} / 100 \mathrm{~mA}$ were available. A scan through the main beam with incident slits set to $500 \mu \mathrm{m}$ gave a fitted instrument resolution of $0.31^{\circ}$. The counting time per point in the specular scatter was $2 \mathrm{~s}$, an entire specular scan being obtained in $20 \mathrm{~min}$. This represents a significant improvement in the data collection rate over polarized neutron experiments.

Tuning the incident energy to just below the Co $L_{3}$ edge resulted in the appearance of a strong, purely magnetic, Bragg peak at half the reciprocal lattice vector of the first structural peak associated with the artificial crystal of the multilayer (Fig. 1). The Kiessig interference fringes, which in reciprocal space have the same phase and periodicity as those measured by hard energy x-ray reflectivity, show that the $\mathrm{x}$-ray wave penetrates to the bottom of the stack $(\sim 1000$ $\AA$ ). Their much lower visibility at the $\mathrm{Cu} L_{3}$ edge arises from the greater absorption at this energy. Mapping the dif-
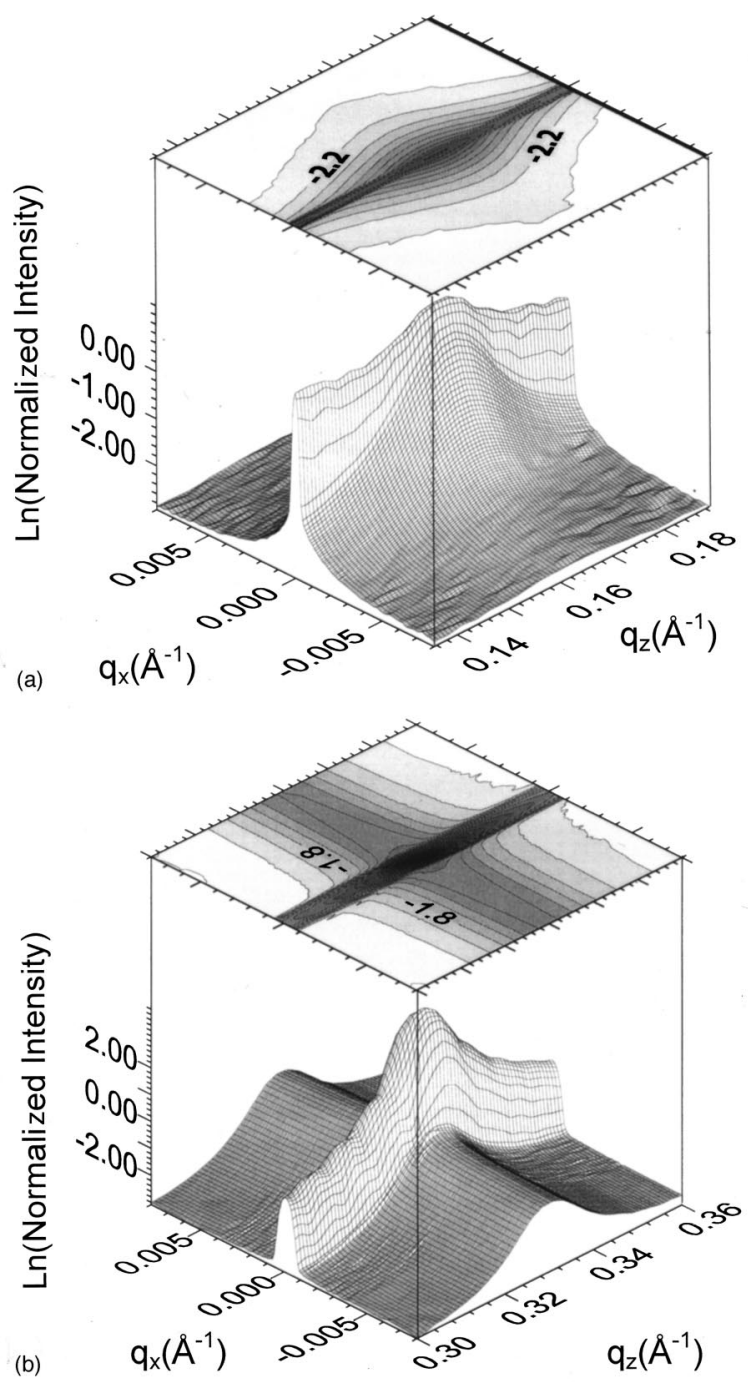

FIG. 2. Full reciprocal space maps of the diffuse scatter around the magnetic (a) and structural (b) Bragg peaks. The contours are in increments of 0.4. The instrument resolved specular ridge is seen in the data as a streak at $q_{x}=0$.

fuse scatter reveals that it is concentrated around the reciprocal lattice points corresponding to the magnetic and charge Bragg peaks (Fig. 2). No such diffuse scatter is found around the position of the magnetic reciprocal-lattice point when off resonance, demonstrating that this is pure magnetic scatter. The extension of the scatter in $q_{x}$ proves that there is a high degree of conformality in both structural and magnetic roughness. The interface roughness of the magnetization thus measured is distinct from the one-dimensional variation of the magnetization as a function of depth through the layer. No magnetic peak was observed at the $\mathrm{Cu} L_{3}$ edge, indicating no detectable polarization of the electrons within the $\mathrm{Cu}$ spacer layer.

The low extension of the magnetic diffuse scatter in $q_{x}$ [Fig. 2(a)] compared with the charge scatter around the structural Bragg peak [Fig. 2(b)] reveals a substantial difference in the in-plane correlation lengths for the two types of roughness. Magnetization of the sample parallel and perpendicular to the scattering plane resulted in a change in the specular magnetic Bragg peak intensity, coupled with changes in the magnetic diffuse scatter distribution in the remanence state 


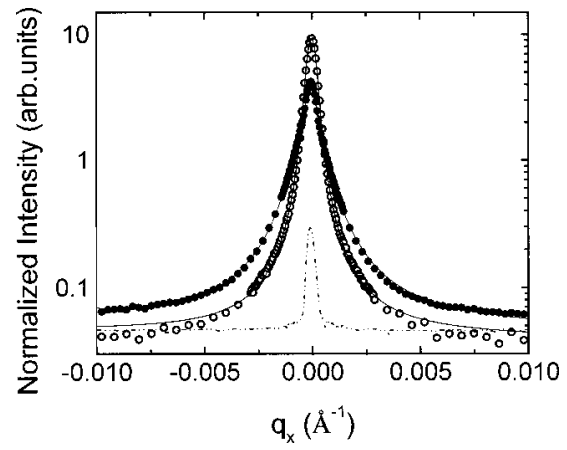

FIG. 3. Remanence-state, transverse scans through the magnetic Bragg peak after magnetization perpendicular (open circles) and parallel (closed circles) to the scattering plane. The sample grown on pure silicon. Also shown is the scan taken at the same momentum transfer as the magnetic Bragg peak with an incident energy 40 $\mathrm{eV}$ below the Co $L_{3}$ edge (dash-dot line).

(Fig. 3). In-field measurements, with field perpendicular to the scattering plane, show a small decrease in the full width at half-height maximum (FWHM) of the $q_{x}$ scan through the magnetic peak. In the two remanence states the correlation length for the sample deposited on pure silicon, determined by fitting a Voigt function, was found to be $9800 \pm 250 \AA$ for magnetization perpendicular and $4600 \pm 250 \AA$ for magnetization parallel to the scattering plane. Both sets of samples showed similar behavior and length scales. Lorentz microscopy reveals a magnetization ripple pattern at all fields, extended perpendicular to the magnetization direction. ${ }^{21}$ The scale of the ripple was comparable with the length scales measured here and its change with field direction agrees with the observed anisotropy. The strong conformality, deduced from the x-ray scattering, is consistent with correlation of this ripple through the strongly coupled multilayer.

In Fig. 3 we show the transverse scan at the magnetic Bragg peak position $\left(q_{z}=0.17 \AA^{-1}\right)$ at an energy $40 \mathrm{eV}$ below the $\mathrm{Co} L_{3}$ absorption edge. Here, and also at the $\mathrm{Cu} L_{3}$ edge, the diffuse charge scatter is extremely low and near the limits of detection. At the Co $L_{3}$ edge, we find similar low levels of diffuse scatter away from the Bragg peak. Scatter around the magnetic Bragg peak thus arises only from conformal roughness with a periodicity of twice the structural period and we can therefore assert that this scatter is purely magnetic in origin, the associated roughness being entirely conformal. The measured amplitude and length scale corresponding to the magnetic roughness are not convolutions of the structural and magnetic interfaces.

Following Freeland et $a l .{ }^{8}$ we have deduced the amplitude of the magnetic roughness within the Born approximation from the ratio of the diffuse and specular scatter integrated over $q_{x}$ through the magnetic Bragg peak. We find that the root mean square (rms) roughness is $3.1 \pm 1 \AA$ when magnetized in the easy direction. Within the experimental error, this is the same as the structural roughness of $2.8 \pm 0.5 \AA$ deduced from the integrated scatter through the structural Bragg peak. This suggests that the direction of magnetization is parallel to the mean surface, the magnetization variation then mapping directly onto the topography. Magnetization of the sample in the hard direction increases the rms magnetic roughness to $5.8 \pm 1 \AA$, greater than the chemical roughness.

Conventional hard $\mathrm{x}$-ray grazing incidence diffuse scatter-

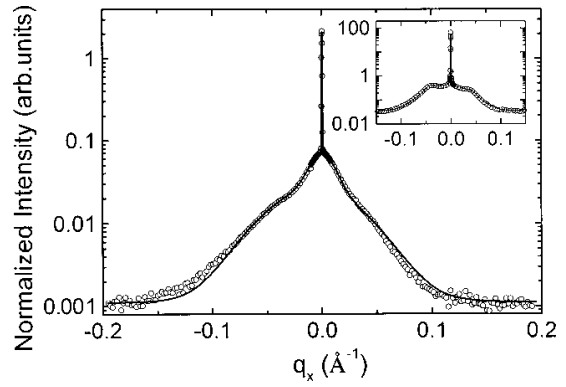

FIG. 4. Transverse scans taken through the structural Bragg peak for the sample deposited on silicon nitride at the Co $L_{3}$ edge. The data (points) and fit (line). Inset: the same scan for the sample deposited on pure silicon showing blazed grating effects.

ing is limited in the range of reciprocal space probed by the critical angle for total external reflection. Using scattering in the incidence plane, only length scales greater than about $1000 \AA$ can be measured directly. This can be circumvented either by fitting the diffuse scatter to that simulated using various models of the interface morphology $y^{3,24}$ or measuring the scattering out of the incidence plane. ${ }^{25}$ The extended range of reciprocal space probed by the soft-x-rays permits the direct measurement of in-plane length scales below 100 $\AA$. We show in Fig. 4 the transverse $\left(q_{x}\right)$ scan through the first structural Bragg peak of a sample grown on a silicon nitride layer. Excluding the spike corresponding to the instrument-resolved specular ridge, the data can be fitted to two Voigt functions with two corresponding correlation lengths $70 \pm 5 \AA$ and $255 \pm 20 \AA$. The inset shows a similar scan for a sample grown on a bare silicon substrate. In addition to a correlation length of circa $72 \pm 10 \AA$, there are two shoulders that are characteristic of a blazed grating. ${ }^{26}$ This corresponds to a step length of $150 \AA$. The two length scales in the different types of sample are similar, the longer being close to the grain size normal to the multilayer, deduced to be $200 \AA$ from high angle diffraction data. No changes were observed in the scattering around the structural peak on application of a magnetic field.

In summary, we have shown that the length scales of the magnetic and structural roughness in $\mathrm{Co} / \mathrm{Cu}$ multilayers differ by one or two orders of magnitude. This is very dramatically different from the results of previous investigations where magnetic and structural length scales are comparable. However, the present experiments genuinely separate the true magnetic diffuse scatter from the diffuse charge scatter and it may be that in the previous experiments, where the magnetic scatter is deduced by subtraction of the scatter on reversal of the magnetization, the interference between these phenomena may result in the short correlation length dominating the data sets.

Magnetic roughness is very strongly correlated through the multilayer, the length scale of the roughness being field dependent and comparable with the size of the magnetic ripple observed in Lorentz electron microscopy. It is small compared with the domain size observed below the coercive field and persists at high fields where Lorentz microscopy shows no domains. The increase in magnetic roughness after magnetization in the hard direction is equivalent to stronger amplitude of ripple. Long correlation length roughness may reduce the strength of the AF coupling through the "orange- 
peel" effect. In these samples, however, this reduction is consistent with orange-peel coupling produced by roughness of a length scale typically $150 \AA{ }^{27}$ which is of the order of the measured structural correlation length. Thus the magnetic roughness can be assumed to play no part in determining the coupling strength. Further, as the in-plane correlation length of the magnetic roughness is so long in comparison with the electron mean free path, it is unlikely that the magnetic roughness measured here is in any way responsible for the spin dependent scatter associated with the GMR. However, we cannot draw any conclusion concerning the effect of magnetic roughness which is identical to the chemical roughness, observed in other multilayer systems.

We wish to thank the staff at the Daresbury SRS, in particular Ian Kirkman and Mark Roper, who provided the diffractometer and station facilities during the taking of the data. Funding from EPSRC is acknowledged.
*Present address: Institut für Festkörperforschung, Forschungszentrum Jülich, 52425 Jülich, Germany.

${ }^{1}$ E. E. Fullerton, D. M. Kelly, J. Guimpel, I. K. Schuller, and Y. Bruynserade, Phys. Rev. Lett. 68, 859 (1992).

${ }^{2}$ D. E. Joyce, C. A. Faunce, P. J. Grundy, B. D. Fulthorpe, T. P. A. Hase, I. Pape, and B. K. Tanner, Phys. Rev. B 58, 5594 (1998).

${ }^{3}$ D. E. Savage, Y.-H. Phang, J. J. Rownd, J. F. MacKay, and M. G. Lagally, J. Appl. Phys. 74, 6158 (1993).

${ }^{4}$ X. Yan and T. Egami, Phys. Rev. B 47, 2362 (1993).

${ }^{5}$ S. K. Sinha, E. B. Sirota, S. Garoff, and H. E. Stanley, Phys. Rev. B 38, 2297 (1988)

${ }^{6}$ M. Wormington, T. P. A. Hase, I. Pape, B. K. Tanner, and D. K. Bowen, Philos. Mag. Lett. 74, 211 (1996).

${ }^{7}$ H. Hashizume, S. Miya, T. Tanaka, N. Ishimatsu, Y. Yamaguchi, N. Hosoito, A. Sakuma, and G. Srajer, Philos. Trans. R. Soc. London, Ser. A 357, 2817 (1999).

${ }^{8}$ J. W. Freeland, V. Chakarian, K. Bussmann, Y. U. Idzerda, H. Wende, and C.-C. Kao, J. Appl. Phys. 83, 6290 (1998).

${ }^{9}$ J. F. McKay, C. Teichert, D. E. Savage, and M. G. Lagally, Phys. Rev. Lett. 77, 3925 (1996).

${ }^{10}$ J. A. Borchers, J. A. Dura, J. Unguris, D. Tulinsky, M. H. Kelley, C. F. Majkrzak, S. Y. Hsu, R. Loloee, W. P. Pratt, Jr., and J. Bass, Phys. Rev. Lett. 82, 2796 (1999).

${ }^{11}$ Y. U. Idzerda, V. Chakarian, and J. W. Freeland, Phys. Rev. Lett. 82, 1562 (1999).

${ }^{12}$ D. Gibbs, D. R. Harshman, E. D. Isaacs, D. B. McWhan, D. Mills and C. Vettier, Phys. Rev. Lett. 61, 1241 (1988); J. P. Hannon, G. T. Trammell, M. Blume, and D. Gibbs, ibid. 61, 1245 (1988).

${ }^{13}$ L. Sève, J. M. Tonnerre, D. Raoux, J. F. Bobo, M. Piecuch, M.
De Santis, P. Troussel, J. M. Brot, V. Chakarian, C. C. Kao, and C. T. Chen, J. Magn. Magn. Mater. 148, 68 (1995).

${ }^{14}$ J. M. Tonnerre, L. Sève, D. Raoux, G. Soullié, B. Rodmacq, and P. Wolfers, Phys. Rev. Lett. 75, 740 (1995).

${ }^{15}$ J. M. Tonnerre, L. Sève, A. Barbara-Dechelette, F. Bartolomé, D. Raoux, V. Chakarian, C. C. Kao, H. Fischer, S. Andrieu, and O. Fruchart, J. Appl. Phys. 83, 6293 (1998).

${ }^{16}$ M. Sacchi, C. F. Hague, E. M. Gullikson, and J. H. Underwood, Phys. Rev. B 57, 108 (1998).

${ }^{17}$ H. Hashizume, N. Ishimatsu, O. Sakata, T. Iizuka, N. Hosoito, K. Namikawa, T. Iwazumi, G. Srajer, C. T. Venkataraman, J. C. Lang, C. S. Nelson, and L. E. Berman, Physica B 248, 133 (1998).

${ }^{18}$ J. P. Hill and D. F. McMorrow, Acta Crystallogr., Sect. A: Found. Crystallogr. A52, 236 (1996).

${ }^{19}$ C. H. Marrows, R. Loloee, and B. J. Hickey, J. Magn. Magn. Mater. 184, 137 (1998).

${ }^{20}$ C. H. Marrows and B. J. Hickey, Phys. Rev. B 59, 463 (1999).

${ }^{21}$ C. H. Marrows, B. J. Hickey, M. Hermann, S. McVitie, and J. N. Chapman, J. Magn. Magn. Mater. (to be published).

${ }^{22} \mathrm{http}: / / \mathrm{www} . \mathrm{dl} \cdot \mathrm{ac} \cdot \mathrm{uk} / \mathrm{SRS} / \mathrm{SXR} / 1-1 \mathrm{hp} /$

${ }^{23} \mathrm{http}: / /$ www.dl.ac.uk/SRS/SXR/5ulhp/

${ }^{24}$ V. Holý and T. Baumbach, Phys. Rev. B 49, 10668 (1994).

${ }^{25}$ T. Salditt, D. Lott, T. H. Metzger, J. Peisl, G. Vignaud, F. Høghøj, O. Schärpf, P. Hinze, and R. Lauer, Phys. Rev. B 54, 5860 (1996).

${ }^{26}$ V. Holý, C. Giannini, L. Tafer, T. Marschner, and W. Stolz, Phys. Rev. B 55, 9960 (1997).

${ }^{27}$ C. H. Marrows, N. Wiser, B. J. Hickey, T. P. A. Hase, and B. K. Tanner, J. Phys.: Condens. Matter 11, 81 (1999). 